\title{
Longitudinal Models for Studying Multivariate Changes and Dynamics
}

\author{
Emilio Ferrer Joseph E. Gonzales \\ Department of Psychology, University of California, Davis, Calif., USA
}

\section{Key Words}

Developmental processes · Dynamic systems · Longitudinal methods · Quantitative analysis · Statistics

\begin{abstract}
In this paper, we describe a longitudinal modeling approach for examining multivariate changes and dynamics. This technique is based on latent change scores and is executed using a structural equation modeling framework. We provide an overview of the model, describing desirable features for identifying dynamics among multiple processes. We then illustrate its application using empirical data consisting of longitudinal processes and conclude the paper with some potential steps for advancing the modeling possibilities.
\end{abstract}

(c) 2014 S. Karger AG, Basel

\section{Introduction}

Over 30 years ago, Baltes and Nesselroade [1] proposed five objectives of longitudinal methodology. These objectives focused on changes at the personal level and included identification of intraindividual changes, in- terindividual differences in intraindividual changes, interrelationships in behavioral changes, causes (determinants) of intraindividual changes and causes (determinants) of interindividual differences in intraindividual changes. Although expressed in the context of psychological processes and development, these principles have a broader scope and are as timely today as when first outlined. In fact, the last two objectives - related to causes, antecedents and sequences - still underlie most fundamental questions in longitudinal research. Moreover, of the many techniques developed over the years, few, if any, can fully achieve these last two objectives.

In this paper, we present a modeling approach centered on changes and dynamics. This modeling approach, based on latent change scores (LCS) [2-4], can be used to examine questions related to Baltes and Nesselroade's [1] last objectives of longitudinal methodology. We first describe this approach, highlighting desirable features for identifying dynamics among multiple processes, then provide a few examples from the extant literature, illustrate the model with an empirical example and conclude with some potential steps for advancing the modeling possibilities.

\section{KARGER}

E-Mail karger@karger.com

www.karger.com/anm
(C) 2014 S. Karger AG, Basel

0250-6807/14/0653-0184\$39.50/0
Emilio Ferrer

Department of Psychology, University of California

One Shields Avenue

Davis, CA 95616-8686 (USA)

E-Mail eferrer@ucdavis.edu 


\section{Description of LCS Models}

Here, we provide a brief description of LCS models. More extensive details are available with regard to mathematical and statistical properties $[2,3,5]$ and comparisons with other models of changes $[3,6,7]$.

LCS models operate on true scores by separating them from measurement errors. Based on a classical true score model, the observed scores of two variables $Y$ and $X$ for person $i$ at time $t$ can be decomposed into true scores $y, x$ and error $e_{y}, e_{x}$, as

$Y_{i t}=y_{i t}+e_{y_{i t}}$ and

$X_{i t}=x_{i t}+e_{x_{i t}}$.

and the true scores can now be used to express the current state of each variable as a function of its previous state plus change, as

$$
\begin{aligned}
& y_{i t}=y_{i t}-1+\Delta_{y_{\mathrm{it}}}, \text { and } \\
& x_{i t}=x_{i t}-1+\Delta_{x_{\mathrm{it}}} .
\end{aligned}
$$

From these equations, the trajectory of the observed variables $Y$ and $X$ for any person $i$ at a given time $t$ can be defined as a function of an initial unobserved score $\left(y_{0}\right.$ and $x_{0}$ ) and the accumulation of changes (i.e. changes in the unobserved variables $\Delta_{y}$ and $\Delta_{x}$ ) up to that time, plus some residuals $\left(e_{y}\right.$ and $\left.e_{x}\right)$, as

$$
\begin{aligned}
& Y_{i t}=y_{i 0}+\left(\sum_{k=1}^{t} \Delta_{y_{i k}}\right)+e_{y_{i t}} \text {, and } \\
& X_{i t}=x_{i 0}+\left(\sum_{k=1}^{t} \Delta_{x_{i k}}\right)+e_{x_{i t}} .
\end{aligned}
$$

This is a general expression that allows multiple specifications for the changes $\left(\Delta_{y}\right.$ and $\left.\Delta_{x}\right)$. One possible specification is as follows

$\Delta_{y_{\mathrm{it}}}=\alpha_{y} \cdot y_{i s}+\beta_{y} \cdot y_{i t-1}+\gamma_{y} \cdot x_{i t-1}$, and

$\Delta_{x_{\mathrm{it}}}=\alpha_{x} \cdot x_{i s}+\beta_{x} \cdot x_{i t-1}+\gamma_{x} \cdot y_{i t-1}$,

where $\alpha$ is the coefficient associated with the additive scores (i.e. slope) $y_{i s}$ and $x_{i s}, \beta$ is a self-feedback parameter representing the influence of the same variable at the previous time $t-1$, and $\gamma$ is a coupling coefficient, representing the influence of the other variable at the previous state $t-1$. A path diagram of a bivariate LCS model is presented in figure 1. This figure presents a system of two variables $Y$ and $X$, measured at $t$ occasions. At each occasion, a latent variable representing changes in the true scores for each variable from the previous occasion $\left(\Delta y_{t}\right.$ and $\left.\Delta x_{t}\right)$ is created. These latent changes are the essential feature of LCS models and are a function of three components: (a) an additive component $\alpha$, typically representing a constant influence on the system; (b) the scores on the same variable at the previous occasion $\beta$, and (c) the scores on the other variable at the previous occasion $\gamma$.
This last component, the coupling parameter, represents forces from one variable at time $t-1$ that lead to changes in the other variable at the next occasion $t$, as the system unfolds over time. These dynamic coefficients are interpreted together, as they jointly bring about the dynamics of the system.

\section{Empirical Example}

The data used in this example come from the 'Motivation in High School Project', a project aimed at examining determinants of motivation among high school students $[6,8]$. The sample comprised 253 volunteer participants (146 males and 107 females) ranging in age from 14 to 18 years (mean: 14.4, SD: 0.84). Students were contacted as they entered high school and followed through their first semester. At four occasions (during the 1st week in class and every 6 weeks since then), students completed a questionnaire about self-perceptions and motivation in their classes.

\section{Perceived Competence}

Perceived competence was measured using the SelfPerception Profile for Adolescents [9]. It contains 5 items and measures the adolescent's perceived ability in various domains. This scale uses a structured alternative response format with scores ranging from 1 (low) to 4 (high). Perceived competence was computed by averaging the scores of the 5 items. The $\alpha$ reliabilities for this scale across the four occasions ranged from 0.83 to 0.86 .

\section{Physical Self-Worth}

Affect toward and evaluation of one's physical self were assessed using the Physical Self-Perception Profile for Adolescents [10]. The scale contains 6 items using a 4-point structured alternative format. One sample item of this scale is: 'Some teenagers are proud of themselves physically BUT other teenagers don't have much to be proud of physically'. The $\alpha$ reliabilities for this scale across the four occasions ranged from 0.85 to 0.88 .

\section{Motivation}

Students assessed their choice of challenging tasks, effort and persistence using an adapted version of TRAAM (the Teacher Rating of Academic Achievement Motivation) [11]. Five items from the original mastery factor were selected to measure the choice of challenging tasks, effort and persistence in the class (e.g. 'Some students prefer easy tasks to more difficult tasks BUT other stu- 


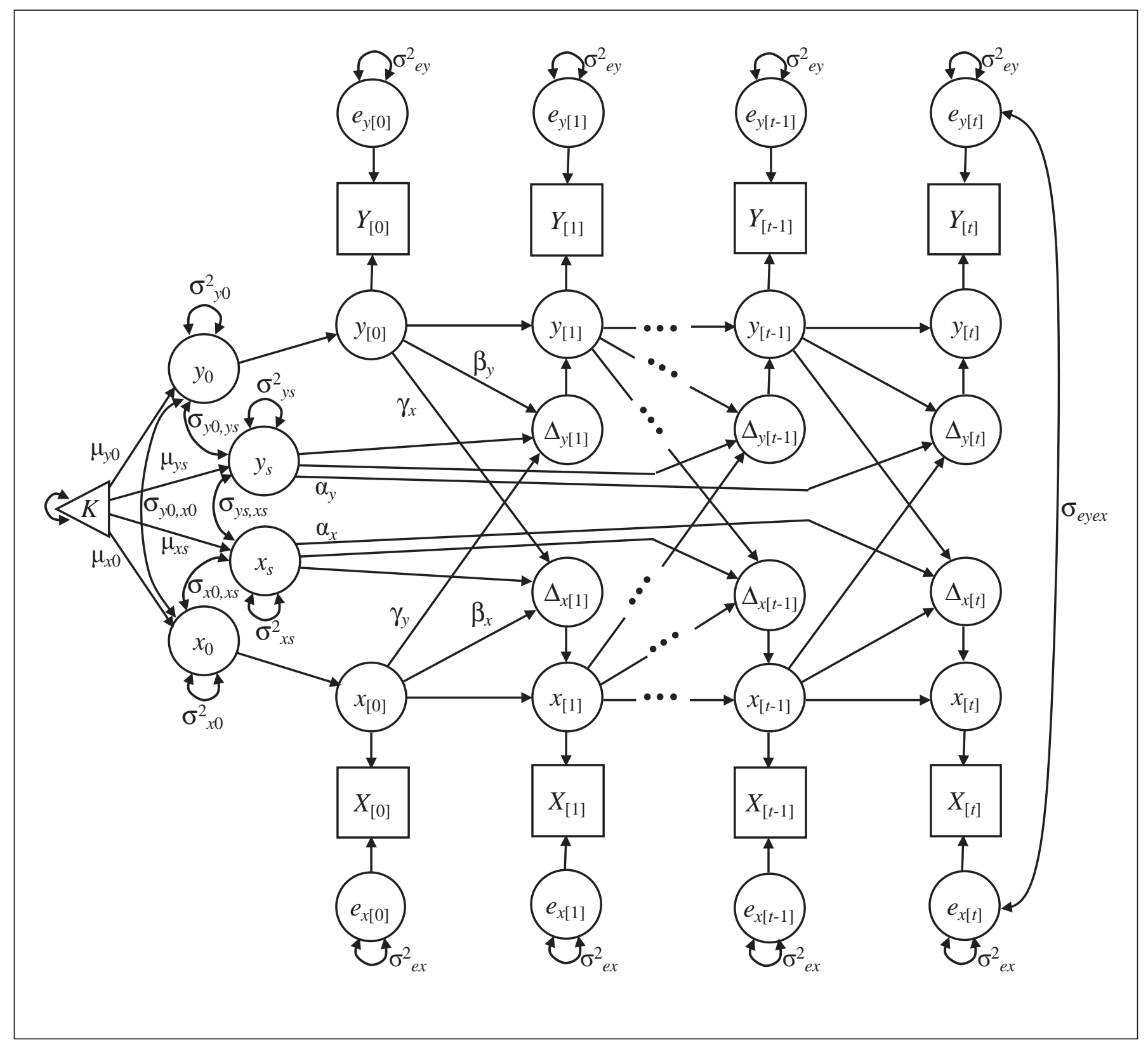

Fig. 1. Path diagram of a bivariate LCS model. Squares represent observed variables, circles represent latent (or unobserved) variables, and the triangle represents a constant. Single-headed arrows indicate regression coefficients, intercepts or means; double-head-

dents prefer more difficult tasks'), with scores ranging from 1 (low) to 4 (high). A composite of motivation was computed by averaging the scores of the 5 items. The $\alpha$ reliabilities for this scale across the four occasions ranged from 0.67 to 0.76 . ed arrows indicate variances and covariances. For each repeated assessment, a latent variable $\left(\Delta_{\mathrm{y}}\right)$ is created to indicate latent changes. These latent changes are the main specification feature of LCS models.

These variables were selected based on the vast literature suggesting a link between individuals' self-perceptions in a given domain and their motivation to engage in activities in that domain $[12,13]$. Similarly, motivation theorists describe perceived competence as a key predictor of participation in activities for intrinsic reasons [14, 
Table 1. Descriptive statistics of variables across the four occasions

\begin{tabular}{lllll}
\hline & Time $1(\mathrm{n}=228)$ & Time $2(\mathrm{n}=201)$ & Time 3 $(\mathrm{n}=210)$ & Time $4(\mathrm{n}=210)$ \\
\hline Perceived competence & $2.76(0.77)$ & $2.86(0.68)$ & $2.93(0.71)$ & $2.96(0.71)$ \\
Physical self-worth & $2.91(0.72)$ & $2.90(0.70)$ & $3.06(0.69)$ & $2.99(0.70)$ \\
Motivation & $2.85(0.55)$ & $2.93(0.55)$ & $2.96(0.61)$ & $2.97(0.61)$ \\
\hline
\end{tabular}

Entries are means (SD). The range for all variables is $1-4$.

Table 2. Parameter estimates and fit statistics from bivariate LCS models

\begin{tabular}{|c|c|c|c|c|c|c|}
\hline & PC & PSW & $\mathrm{PC}$ & MOT & PSW & MOT \\
\hline \multicolumn{7}{|l|}{ Fixed parameters } \\
\hline Initial mean $\mu_{0}$ & $2.75(0.05)$ & $2.88(0.04)$ & $2.75(0.05)$ & $2.83(0.03)$ & $2.89(0.04)$ & $2.83(0.03)$ \\
\hline Proportion $\beta$ & $-1.22(0.18)$ & $-0.08(0.06)^{?}$ & $-0.49(0.22)$ & $-0.18(0.24)^{?}$ & $-1.01(0.64)^{?}$ & $-0.21(0.34)^{?}$ \\
\hline Coupling $\gamma$ & $1.04(0.18)$ & $0.01(0.05)^{?}$ & $0.45(0.19)$ & $0.12(0.11)^{?}$ & $-0.72(0.50)^{?}$ & $0.09(0.13)^{?}$ \\
\hline Additive variance $\sigma_{s}$ & $0.18(0.06)$ & $0.02(0.01)$ & $0.05(0.04)^{?}$ & $0.01(0.01)^{?}$ & $0.34(0.39)^{?}$ & $0.02(0.03)^{?}$ \\
\hline Residual $\sigma_{0}$ & $0.10(0.01)$ & $0.11(0.01)$ & $0.11(0.01)$ & $0.09(0.01)$ & $0.10(0.01)$ & $0.10(0.01)$ \\
\hline \multicolumn{7}{|l|}{ Model fit statistics } \\
\hline$\chi^{2}$ (d.f.) & & $67.7(25)$ & & $41.3(25)$ & & $47.1(25)$ \\
\hline BIC & & 2,282 & & 2,238 & & 2,296 \\
\hline $\mathrm{BIC}$ & & 2,293 & & 2,234 & & 2,293 \\
\hline RMSEA & & 0.092 & & 0.054 & & 0.063 \\
\hline$\Delta \chi^{2} / \Delta$ d.f. & & $22.2(2)$ & & $6.3(2)$ & & $7.7(2)$ \\
\hline
\end{tabular}

Entries are means (SD). $N_{\text {data points }}=1,698$. The range for all variables is $1-4$. $\mathrm{PC}=$ Perceived competence; PSW = physical self-worth; MOT $=$ motivation; BIC = Bayesian information criterion; RMSEA = root mean square error of approximation; ? = parameter estimate whose 95\% bootstrap confidence intervals contain zero. The parameters are defined at $t=0$ and for Dt $=1$ measurement occasion. All parameters are full maximum likelihood estimates.

15]. A consequence of intrinsic motivation is the choice of challenging tasks, effort and persistence in the face of failures. In our analyses, we examined the dynamics among competence, physical self-worth and motivation over time with the goal of identifying the mechanisms underlying such dynamics.

Table 1 reports descriptive statistics for all variables across the four measurement occasions. These statistics indicate a slight increase in all constructs across time. The subsequent analyses are aimed at investigating the growth in each variable as well as the dynamic interrelations among them.

Longitudinal Dynamic Models

\section{Results}

\section{Bivariate LCS Models}

We began by fitting a series of bivariate LCS models. Focusing on the dynamics between 2 variables at a time is advantageous for two reasons. First, it allows us to investigate the dynamic interactions of two processes, which is less complex than other multivariate systems. Second, it can serve as a starting point for assessing the dynamics in other more complex systems, such as that with three processes. Results from these initial analyses are presented in table 2 , and they describe the interrela- 


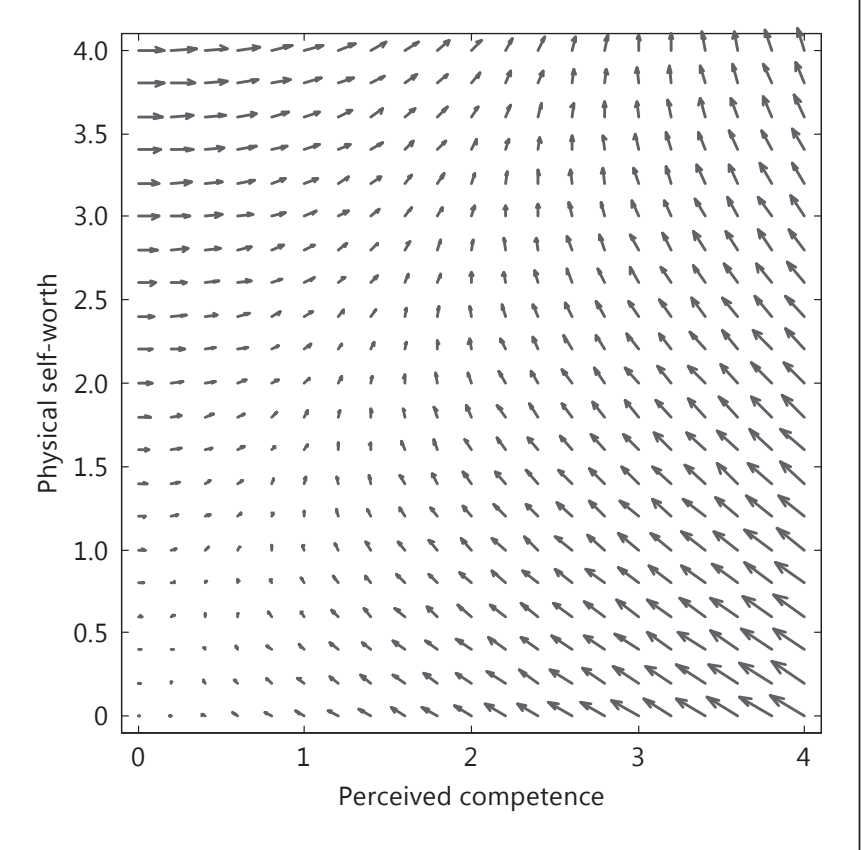

Fig. 2. Vector field for perceived competence and physical selfworth latent scores.

tions between perceived competence and physical selfworth, perceived competence with motivation and physical self-worth with motivation.

Perceived Competence and Physical Self-Worth. Estimates from the perceived competence and physical selfworth model indicate that there was a positive additive mean for both perceived competence and physical selfworth ( $\alpha=0.48$ and 0.28 , respectively), representing a constant amount of change per measurement occasion. Furthermore, there were negative lagged $(t-1)$ effects of prior perceived competence scores $(\beta=-1.22)$ and positive coupling effects of prior physical self-worth scores $(\gamma=1.04)$ on change in perceived competence. In contrast, neither prior physical self-worth scores $(\beta)$ nor perceived competence scores $(\gamma)$ were related to changes in self-worth.

These results suggest that changes in perceived competence are negatively influenced by a latent additive process, a lagged relation from its own preceding score and a lagged relation from the preceding score of self-worth. However, change in physical self-worth is entirely driven by a latent additive process. To test whether inclusion of the coupling parameter estimates should be part of the model, we constrained the coupling estimates to equal zero. We observed a significant increase in model misfit

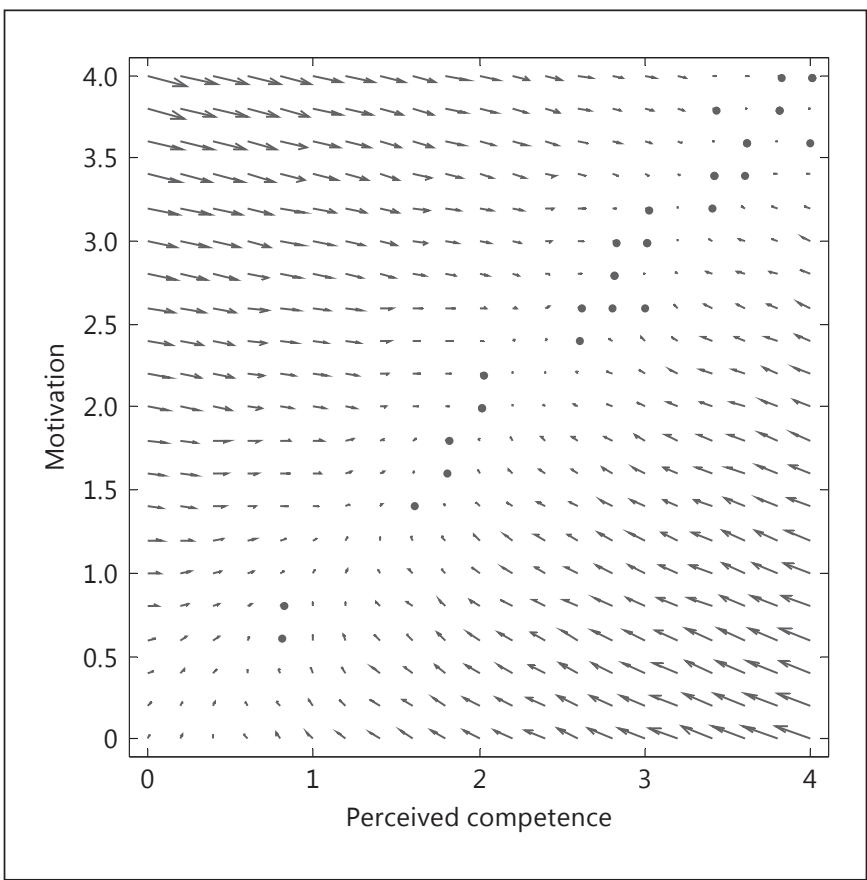

Fig. 3. Vector field for perceived competence and motivation latent scores.

in our constrained model $\left(\Delta \chi^{2}=22.2, \Delta\right.$ d.f. $\left.=2, \mathrm{p}<0.01\right)$. However, when only the nonsignificant coupling was constrained to be zero, the model fit was not altered, suggesting that the coupling from perceived competence to physical self-worth was not necessary.

A statistical vector field (fig. 2) depicts the expected changes in perceived competence and physical self-worth from one time point to the next given a set of scores on both variables. For example, for scores of about 2 for perceived competence and 3 for self-worth, the expected changes are positive for both variables. This graph depicts an attractor-like feature running along the positive diagonal. Given scores in this area, the expected changes are positive, albeit small, for both constructs.

Perceived Competence and Motivation. Estimates from the perceived competence and motivation model indicate that there is no significant effect of an additive mean $(\alpha)$ for changes in either perceived competence or motivation. However, changes in perceived competence were negatively related to lagged perceived competence scores $(\beta=-0.49)$ and positively related to lagged motivation scores $(\gamma=0.45)$. In contrast, change in motivation was not significantly related to lagged motivation or perceived competence scores, implying that motivation scores do not change over time in this model. 


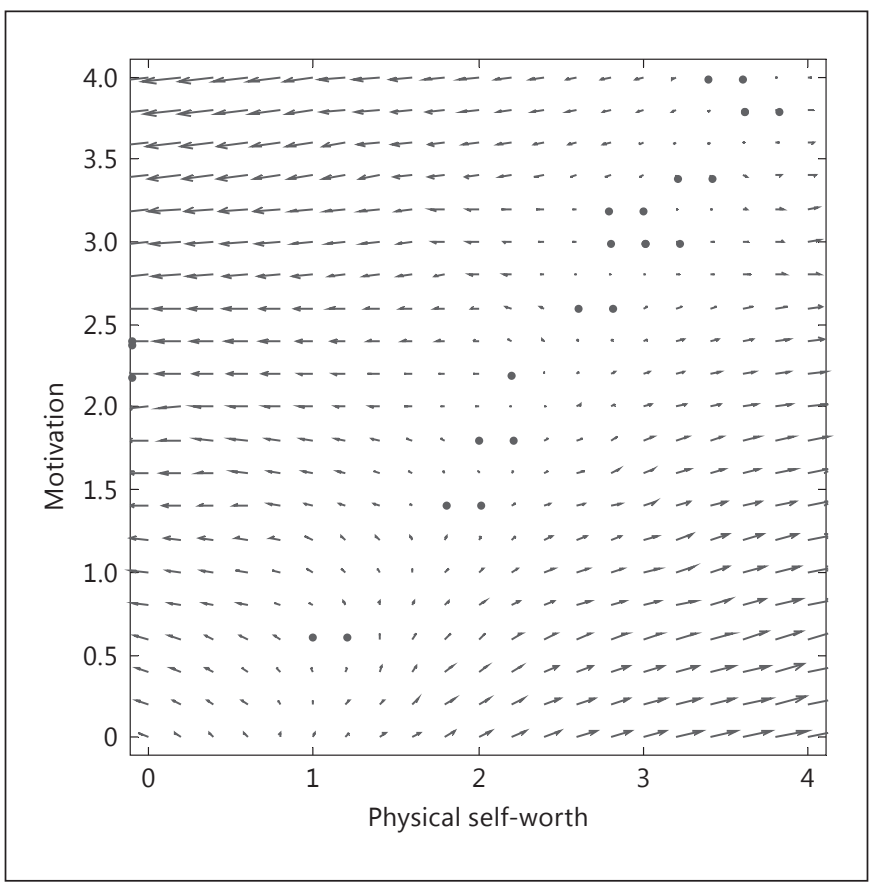

Fig. 4. Vector field for physical self-worth and motivation latent scores.

To test whether inclusion of the coupling parameter estimates should be part of the model, we constrain the coupling estimates to equal zero. We observe a significant yet small increase in model misfit $\left(\Delta \chi^{2}=6.3, \Delta\right.$ d.f. $=2$, $\mathrm{p}=0.04)$ as a result of this constraint. When only the nonsignificant coupling from perceived competence to motivation was constrained to be zero, the model fit did not worsen significantly.

These relations are depicted using a statistical vector field (fig. 3). The lack of change in motivation is evident as there is no movement along the motivation axis regardless of where the latent scores of perceived competence and motivation are located. However, large scores in perceived competence and low scores in motivation (bottom right of the plot) result in negative changes for perceived competence. In contrast, larger motivation scores and lower perceived competence scores result in positive changes for perceived competence. Finally, when motivation scores are approximately half of the perceived competence scores there is little to no change in either variable.

Physical Self-Worth and Motivation. Estimates from the physical self-worth and motivation model indicate that there is no significant effect of an additive mean $(\alpha)$ for either physical self-worth or motivation, nor is there a significant effect of lagged physical self-worth or motivation scores on change scores for either variable. These parameter estimates suggest that there is no change in either latent score over time.

In this instance, it is reasonable to expect that the estimation of the coupling parameters is unnecessary due to nonsignificance. However, when we constrain the coupling estimates to equal zero in the model, we observe a significant - yet small - increase in model misfit $\left(\Delta \chi^{2}=\right.$ 7.7, $\Delta$ d.f. $=2, \mathrm{p}=0.02$ ). Given the model estimates for physical self-worth and motivation, it is not surprising to observe the lack of change depicted in a statistical vector field of this model (fig. 4).

\section{Multivariate LCS Models}

Results from the bivariate analyses indicate that change in physical self-worth was unrelated to lagged scores of motivation and perceived competence, whereas change in motivation was unrelated to lagged scores of personal self-worth and perceived competence. This suggests that neither process is better explained when modeled with each other as a single system. However, changes in perceived competence were significantly related to lagged scores of both physical self-worth and motivation. As a result of these coupling effects, it may be best to model these three processes simultaneously as a single multivariate system.

Changes Predicted by Prior Levels. A trivariate LCS model was used to describe changes in perceived competence, physical self-worth and motivation as well as their interrelations as a single system. In this model, each process (e.g. perceived competence) has two coupling parameter estimates representing lagged $(t-1)$ influences from the other two variables in the system (e.g. physical self-worth and motivation; fig. 5) in addition to lagged effects from itself and an additive mean. The goal of this model is to examine the dynamics underlying changes and interrelations among the three processes simultaneously as a single multivariate system $[16,17]$. A general expression of the changes underlying this multivariate system can be specified as follows

$$
\begin{aligned}
& \Delta_{y_{\mathrm{it}}}=\alpha_{y} \cdot y_{i s}+\beta_{y} \cdot y_{i t-1}+\gamma_{y x} \cdot x_{i t-1}+\gamma_{y z} \cdot z_{i t-1}, \\
& \Delta_{x_{\mathrm{it}}}=\alpha_{x} \cdot x_{i s}+\beta_{x} \cdot x_{i t-1}+\gamma_{x y} \cdot y_{i t-1}+\gamma_{x z} \cdot z_{i t-1}, \text { and } \\
& \Delta_{z_{\mathrm{it}}}=\alpha_{z} \cdot z_{i s}+\beta_{z} \cdot z_{i t-1}+\gamma_{z y} \cdot y_{i t-1}+\gamma_{z x} \cdot x_{i t-1} .
\end{aligned}
$$

Table 3 presents the parameter estimates from this multivariate model. These estimates indicate that there is no significant effect of an additive mean $(\alpha)$ for any of the processes. There was a significant lagged effect of perceived competence scores $(\beta=-1.23)$ on its own changes, but this effect was not evident for physical self-worth or 


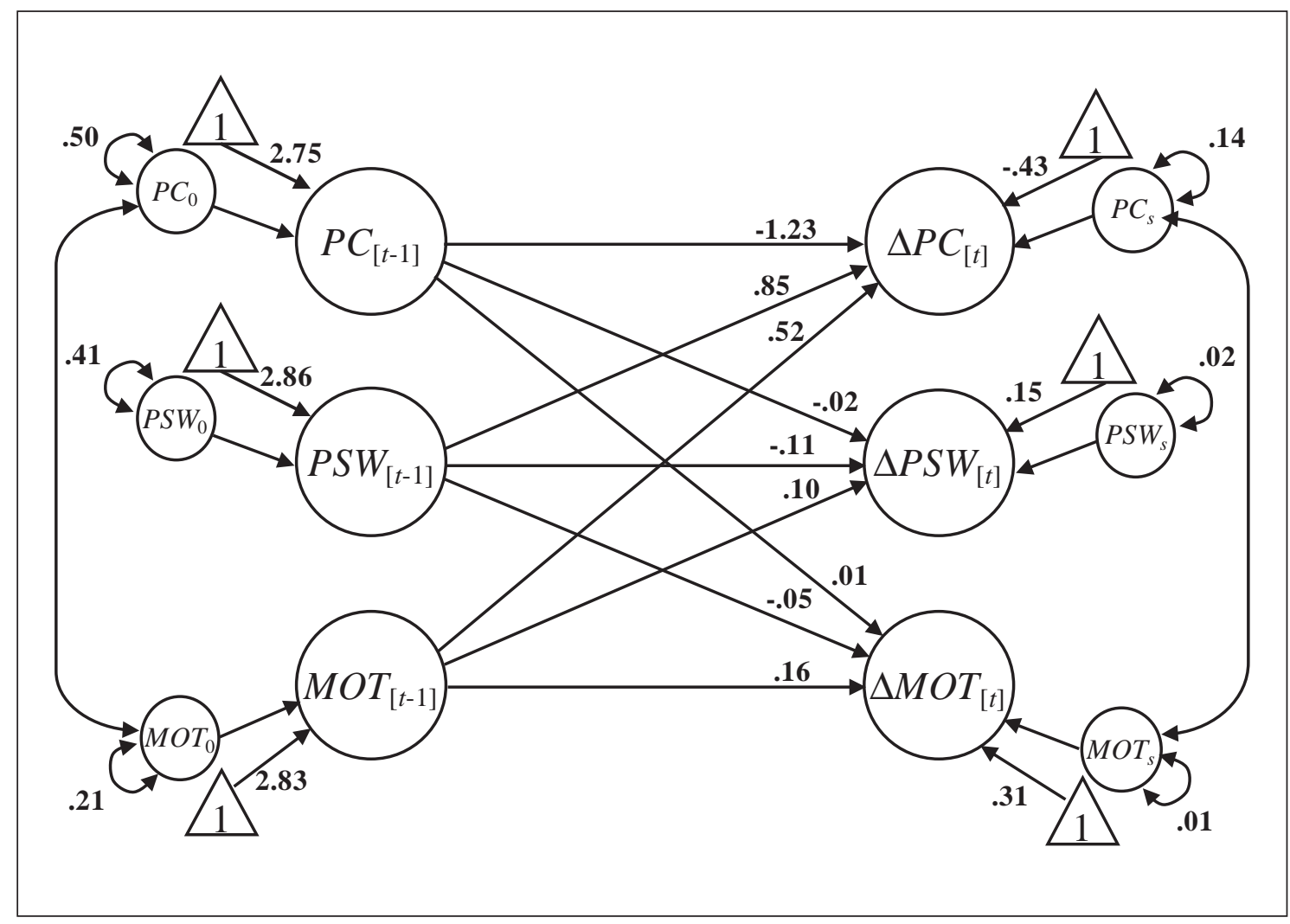

Fig. 5. Representation of the dynamics of a multivariate system. $\mathrm{PC}=$ Perceived competence; $\mathrm{PSW}=$ physical self-worth; $\mathrm{MOT}=$ motivation; $P C_{[t-1]}=$ perceived competence scores at time $t-1$; $\Delta P C_{[t]}=$ changes in perceived competence at time $t$; triangle $=$ con- stant $(=1)$; path from constant to score $[t-1]=$ initial level; path from constant to change $[t]=$ linear additive component; $P C_{0}=$ variance of initial level scores; $P C_{s}=$ variance of the linear additive component. motivation. Changes in perceived competence were related to prior physical self-worth and motivation scores $(\gamma=0.85$ and 0.52 , respectively). Additionally, changes in physical self-worth were related to prior motivation scores $(\gamma=0.10)$ but not perceived competence. Finally, no coupling parameters leading to changes in motivation were apparent.

To evaluate if estimation of the coupling parameters was necessary, the model was fit with these parameters for all variables constrained to equal zero. This model showed a significant increase in misfit $\left(\Delta \chi^{2}=31.9, \Delta\right.$ d.f. $=6, p<$ 0.01 ). When only the nonsignificant coupling parameters were constrained to zero, the change in model fit was not significant suggesting that such couplings are not necessary to account for the data.

Model parameter estimates suggest that changes in perceived competence are driven by its own prior scores, as well as prior scores of both physical self-worth and motivation. In contrast, physical self-worth changes are only influenced by prior scores from motivation. Finally, according to this model, motivation scores show no changes over time. In comparison to the previous bivariate models, these lagged relations are very similar, with the exception of the relation of prior motivation scores on changes in perceived self-worth.

Figure 5 displays the implied dynamics of this multivariate system. This representation depicts the complexity of such dynamics, with multiple forces operating simultaneously. One possible way to identify relevant information from this system is to focus on the changes in one particular variable. For example, individuals' perceived competence scores start at 2.75 at the first measurement occasion, with a variance of 0.50 . At each subsequent occasion, individuals' changes in perceived competence are positively related to scores on physical self-worth and motivation from the prior time point $(\gamma=$ 0.85 and 0.52 , respectively) but also negatively predicted by perceived competence scores from the previous occa- 
Table 3. Parameter estimates and fit statistics from a trivariate LCS model

\begin{tabular}{|c|c|c|c|}
\hline & PC & PSW & MOT \\
\hline \multicolumn{4}{|l|}{ Fixed parameters } \\
\hline Initial mean $\mu_{0}$ & $2.75(0.05)$ & $2.86(0.05)$ & $2.83(0.03)$ \\
\hline Additive mean $\alpha$ & $-0.43(0.23)^{?}$ & $0.15(0.10)^{?}$ & $-0.31(0.17)^{?}$ \\
\hline Proportion $\beta$ & $-1.23(0.16)$ & $-0.11(0.07)^{?}$ & $0.16(0.09)^{?}$ \\
\hline \multicolumn{4}{|l|}{ Coupling $\gamma$} \\
\hline$P C \rightarrow$ & - & $-0.02(0.05)^{?}$ & $0.01(0.04)^{?}$ \\
\hline$P S W \rightarrow$ & $0.85(0.15)$ & - & $-0.05(0.04)^{?}$ \\
\hline$M O T \rightarrow$ & $0.52(0.11)$ & $0.10(0.04)$ & - \\
\hline \multicolumn{4}{|l|}{ Variance parameters } \\
\hline Initial variance $\sigma_{0}$ & $0.50(0.06)$ & $0.41(0.05)$ & $0.21(0.03)$ \\
\hline Additive variance $\sigma$ s & $0.14(0.04)$ & $0.02(0.01)$ & $0.01(0.01)^{?}$ \\
\hline Residual $\sigma_{0}$ & $0.10(0.01)$ & $0.11(0.01)$ & $0.10(0.01)$ \\
\hline \multicolumn{4}{|l|}{ Model fit statistics } \\
\hline$\chi^{2}$ (d.f.) & & $105.2(57)$ & \\
\hline BIC & & 3,225 & \\
\hline RMSEA & & 0.057 & \\
\hline \multicolumn{4}{|l|}{ No $\gamma$} \\
\hline$\chi^{2}$ (d.f.) & & $137.1(63)$ & \\
\hline $\mathrm{BIC}$ & & 3,234 & \\
\hline RMSEA & & 0.067 & \\
\hline$\Delta \chi^{2} / \Delta$ d.f. & & $31.9(6)$ & \\
\hline
\end{tabular}

Entries are means (SD). $N_{\text {data points }}=2,547$. The range for all variables is $1-4$. $\mathrm{PC}=$ Perceived competence; PSW = physical self-worth; MOT = motivation; BIC = Bayesian information criterion; RMSEA = root mean square error of approximation; ${ }^{?}=$ parameter estimate whose $95 \%$ bootstrap confidence intervals contain zero. The parameters are defined at $t=0$ and for $\mathrm{Dt}=1$ measurement occasion. All parameters are full maximum likelihood estimates. sion $(\beta=-1.23)$. Similarly, one can focus on the forces that a particular variable exerts in the system. For example, perceived competence negligibly predicts changes in both physical self-worth and motivation $(\gamma=-0.02$ and 0.01 , respectively) but is negatively related to its own subsequent changes $(\beta=-1.23)$.

Changes Predicted by Prior Levels and Prior Changes. As an alternative to the previous trivariate LCS model, we fit a model in which changes in each process were predicted by the prior scores in itself and the other variables in the model, as well as prior changes $[7,18,19]$ (see fig. 6 for a bivariate version). This model differs from the previous model in that changes in a given process (e.g. $\left.\Delta_{y}\right)$ are not only predicted by scores at the previous occasion (itself, $\beta_{1 y}$, and other variables, $\gamma_{1 y}$ ) but also by prior changes (itself, $\beta_{2 y}$, and other variables, $\gamma_{1 y}$ ), in addition to a latent additive effect $\left(\alpha_{y}\right)$. A general specification of the changes for this multivariate system can be expressed as follows:

Longitudinal Dynamic Models

$$
\begin{aligned}
& \quad \Delta_{y_{\mathrm{it}}}=\alpha_{y} \cdot y_{i s}+\beta_{y 1} \cdot y_{i t-1}+\beta_{y 2} \cdot \Delta y_{i t-1}+\gamma_{y x 1} \cdot x_{i t-1}+\gamma_{y x 2} \cdot \Delta x_{i t-1} \\
& +\gamma_{y z 1} \cdot z_{i t-1}+\gamma_{y z 2} \cdot \Delta z_{i t-1}, \\
& \Delta_{x_{\mathrm{it}}}=\alpha_{x} \cdot x_{i s}+\beta_{x 1} \cdot x_{i t-1}+\beta_{x 2} \cdot \Delta x_{i t-1}+\gamma_{x y 1} \cdot y_{i t-1}+\gamma_{x y 2} \cdot \Delta y_{i t-1} \\
& +\gamma_{x z 1} \cdot z_{i t-1}+\gamma_{x z 2} \cdot \Delta z_{i t-1}, \\
& \Delta_{z_{\mathrm{it}}}=\alpha_{z} \cdot z_{i s}+\beta_{z 1} \cdot z_{i t-1}+\beta_{z 2} \cdot \Delta z_{i t-1}+\gamma_{z y 1} \cdot y_{i t-1}+\gamma_{z y 2} \cdot \Delta y_{i t-1}+ \\
& \gamma_{z x 1} \cdot x_{i t-1}+\gamma_{z x 2} \cdot \Delta x_{i t-1} .
\end{aligned}
$$

Parameter estimates from this new specification are reported in table 4 . Changes in perceived competence were related to an additive mean $(\alpha=-0.65)$, an autoregression effect from prior perceived competence scores $\left(\beta_{1}=-1.36\right)$ and coupling effects from both perceived self-worth and motivation $\left(\gamma_{1}=0.99\right.$ and 0.56 , respectively). In addition, such changes in perceived competence were also related to their own changes at the prior occasion $\left(\beta_{2}=1.21\right)$ but not to prior changes in perceived self-worth or motivation.

For perceived self-worth, the additive mean effect was not different from zero, which was consistent with the previous trivariate model. However, changes in perceived self-worth were related to previous levels of self-worth 
Table 4. Parameter estimates and fit statistics from a trivariate alternative LCS model

\begin{tabular}{|c|c|c|c|}
\hline & PC & PSW & MOT \\
\hline \multicolumn{4}{|l|}{ Fixed parameters } \\
\hline Initial mean $\mu_{0}$ & $2.77(0.05)$ & $2.91(0.05)$ & $2.84(0.04)$ \\
\hline Additive mean $\alpha$ & $-0.65(0.31)$ & $-0.01(0.13)^{?}$ & $-0.59(0.27)$ \\
\hline \multicolumn{4}{|l|}{ Proportion $\beta$} \\
\hline$y_{t-1} \rightarrow$ & $-1.36(0.35)$ & $-0.11(.06)$ & $0.09(0.09)^{?}$ \\
\hline$\Delta y_{t-1} \rightarrow$ & $1.21(0.56)$ & $-0.76(0.62)^{?}$ & $-0.61(0.28)$ \\
\hline \multicolumn{4}{|l|}{ Coupling $\gamma$} \\
\hline$P C_{t-1} \rightarrow$ & - & $-0.00(0.06)^{?}$ & $0.08(0.08)^{?}$ \\
\hline$P S W_{t-1} \rightarrow$ & $0.99(0.31)$ & - & $0.05(0.05)^{?}$ \\
\hline $\mathrm{MOT}_{t-1} \rightarrow$ & $0.56(0.14)$ & $0.11(0.05)$ & - \\
\hline$\Delta P C_{t-1} \rightarrow$ & - & $1.82(0.69)$ & $0.13(0.33)^{?}$ \\
\hline$\Delta P S W_{t-1} \rightarrow$ & $-0.33(0.42)^{?}$ & - & $0.15(0.21)^{?}$ \\
\hline$\Delta \mathrm{MOT}_{t-1} \rightarrow$ & $-0.09(0.51)^{?}$ & $0.81(0.73)^{?}$ & - \\
\hline \multicolumn{4}{|l|}{ Variance parameters } \\
\hline Initial variance $\sigma_{0}$ & $0.41(.05)$ & $0.41(0.04)$ & $0.20(0.03)$ \\
\hline Additive variance $\sigma_{s}$ & $0.18(.10)$ & $0.01(0.01)^{?}$ & $0.02(0.01)$ \\
\hline Residual $\sigma_{0}$ & $0.11(.01)$ & $0.10(0.01)$ & $0.10(0.01)$ \\
\hline \multicolumn{4}{|l|}{ Model fit statistics } \\
\hline$\chi^{2}$ (d.f.) & & $125.9(49)$ & \\
\hline BIC & & 3,290 & \\
\hline RMSEA & & 0.078 & \\
\hline
\end{tabular}

Entries are means (SD). $N_{\text {data points }}=2,547$. Range for all variables is $1-4$. PC $=$ Perceived competence; PSW = physical self-worth; MOT = motivation; BIC = Bayesian information criterion; RMSEA = root mean square error of approximation; ${ }^{\text {? }}=$ parameter estimate whose $95 \%$ bootstrap confidence intervals contain zero. The parameters are defined at $t=0$ and for $\mathrm{D}_{\mathrm{t}}=1$ measurement occasion. All parameters are full maximum likelihood estimates. $\left(\beta_{1}=-0.11\right)$, as well as to coupling effects from motivation $\left(\gamma_{1}=0.11\right)$ and changes in perceived competence $\left(\gamma_{2}=\right.$ 1.82 , respectively). In contrast, changes in motivation were predicted by an additive mean effect $(\alpha=-0.59)$ and by their own changes at the prior occasion $\left(\beta_{2}=-0.61\right)$, but not from the other variables in the system.

Model-Implied Trajectories for the Multivariate System. To identify the model-implied trajectories for each variable in the system, we generated starting scores and applied the model estimates in order to produce change trajectory expectations. We carried out this approach for each of the variables in the trivariate model. Results from this approach are displayed in figure 7 . Here, we depict expected latent trajectories for each of the variables based on the two multivariate models (solid and dashed lines, respectively). As expected, there is a difference in the trajectories for motivation between the first and second trivariate model. This is unsurprising since the first model estimated no change in this variable. However, other differences in the mean change trajectories are apparent between both models.
For example, when using estimates from the second multivariate model, perceived competence shows an asymptotic trajectory of change after the second observation, and this is true across different starting values of physical self-worth and motivation (fig. 8b). This pattern, however, is not observed when evaluating the trajectories generated from the first multivariate model (fig. 8a). Similarly, when using the first model to project change in physical self-worth, we observe three distinct linear trajectories making a fan shape over time with the upper and lower values being driven by minimum versus maximum motivation scores (fig. 9a). When we evaluate projections for the same variable using the second model, we see greater divergence in trajectories and a loss of the distinct fan pattern (fig. 9b).

Finally, when evaluating projected motivation change trajectories, the range of the scale needs to be reduced in order to observe any actual variability between the first and second multivariate models (fig. 10). This is due to the relatively small rate of change in motivation scores over time. While there appear to be several patterns of 


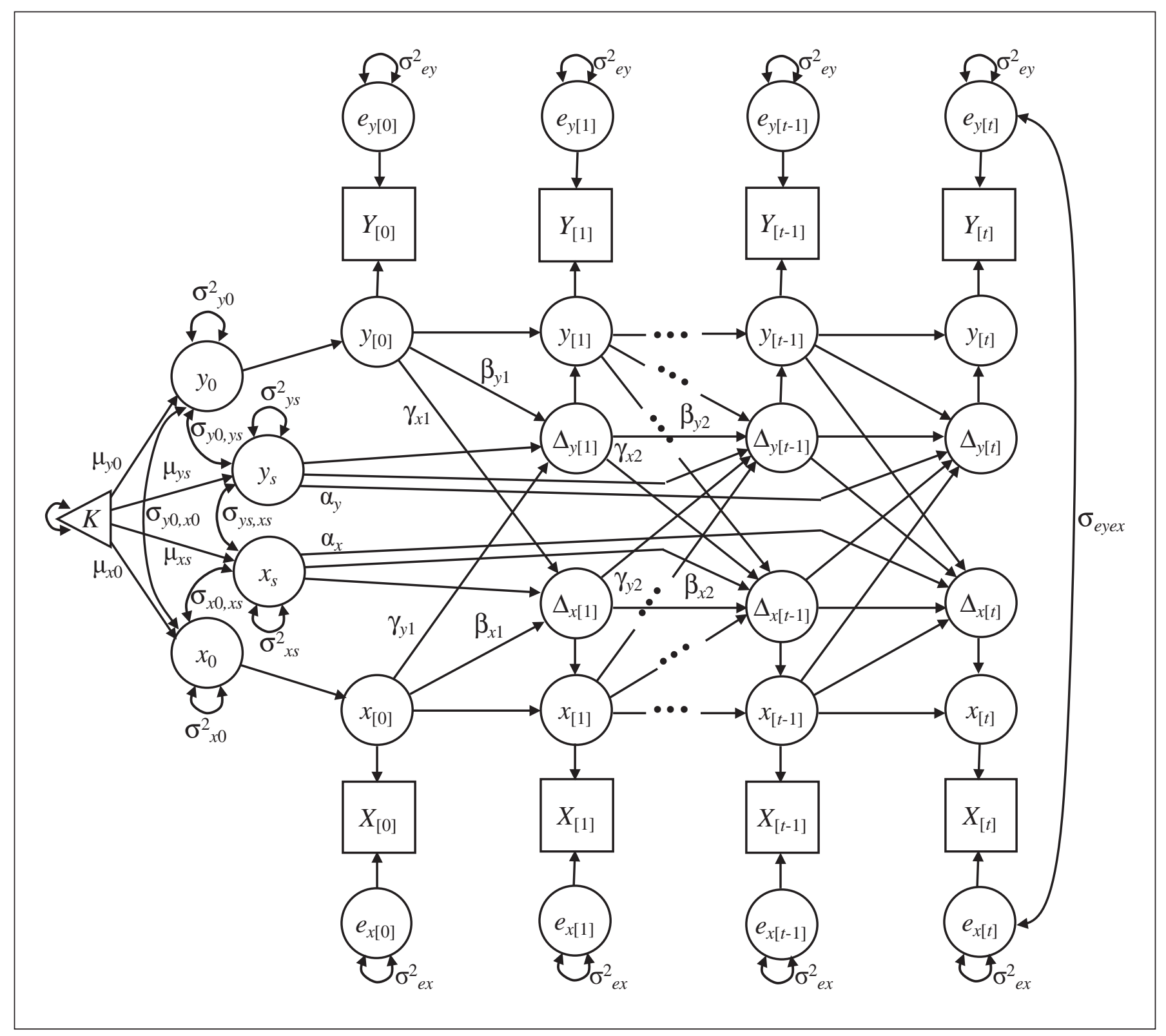

Fig. 6. Path diagram of a bivariate LCS model with effects from previous changes. For further information, see legend to figure 1.

change for motivation as a function of perceived competence and physical self-worth, this is in fact a result of the variability in the additive mean and proportion of prior change in motivation. That is, there is such a small amount of actual change that the variability in the additive mean and proportion estimates, by chance, resulted in what appears to be divergent trajectories of change. As a result, it is best to focus interpretation of model differences on the mean trajectory of change for motivation using the second model.

\section{Discussion}

In this paper, we describe a longitudinal modeling approach for examining multivariate changes and dynamics. This technique is based on LCS and is executed using a structural equation modeling (SEM) framework. As such, this modeling approach allows for the investigation of many different hypotheses about changes in one or multiple variables. Examples of this broad scope include the examination of pretest-posttest changes in random- 
Fig. 7. Expected latent trajectories for each of the variables based on the first (solid line) and second (dashed line) multivariate model.

Fig. 8. Expected latent trajectories for perceived competence as a function of varying initial scores (minimum, average and maximum) for physical self-worth (PSW) and motivation (MOT) based on the first (a) and second (b) multivariate model.
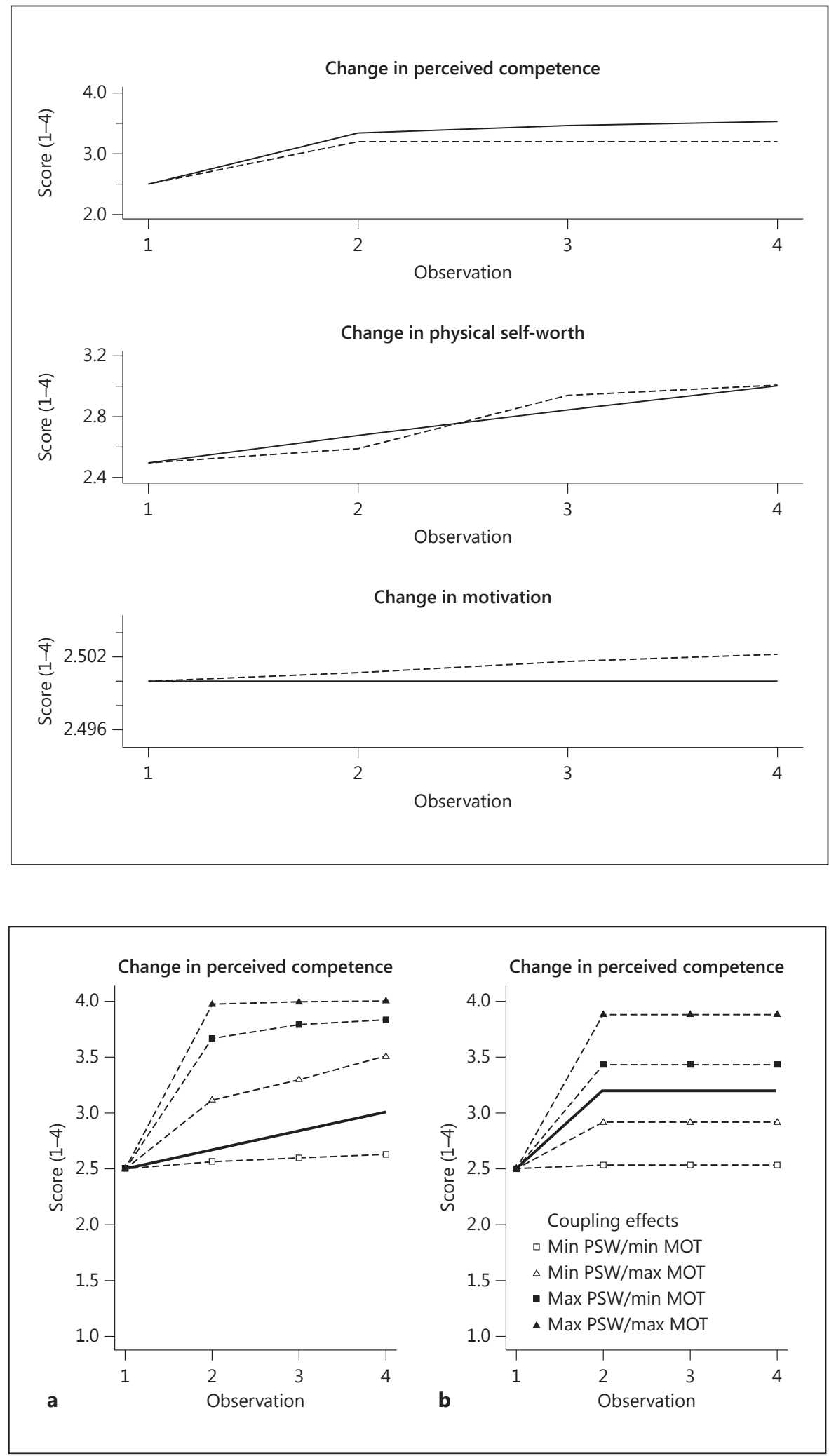
Fig. 9. Expected latent trajectories for physical self-worth as a function of varying initial scores (minimum, average and maximum) for perceived competence (PC) and motivation (MOT) based on the first (a) and second (b) multivariate model.
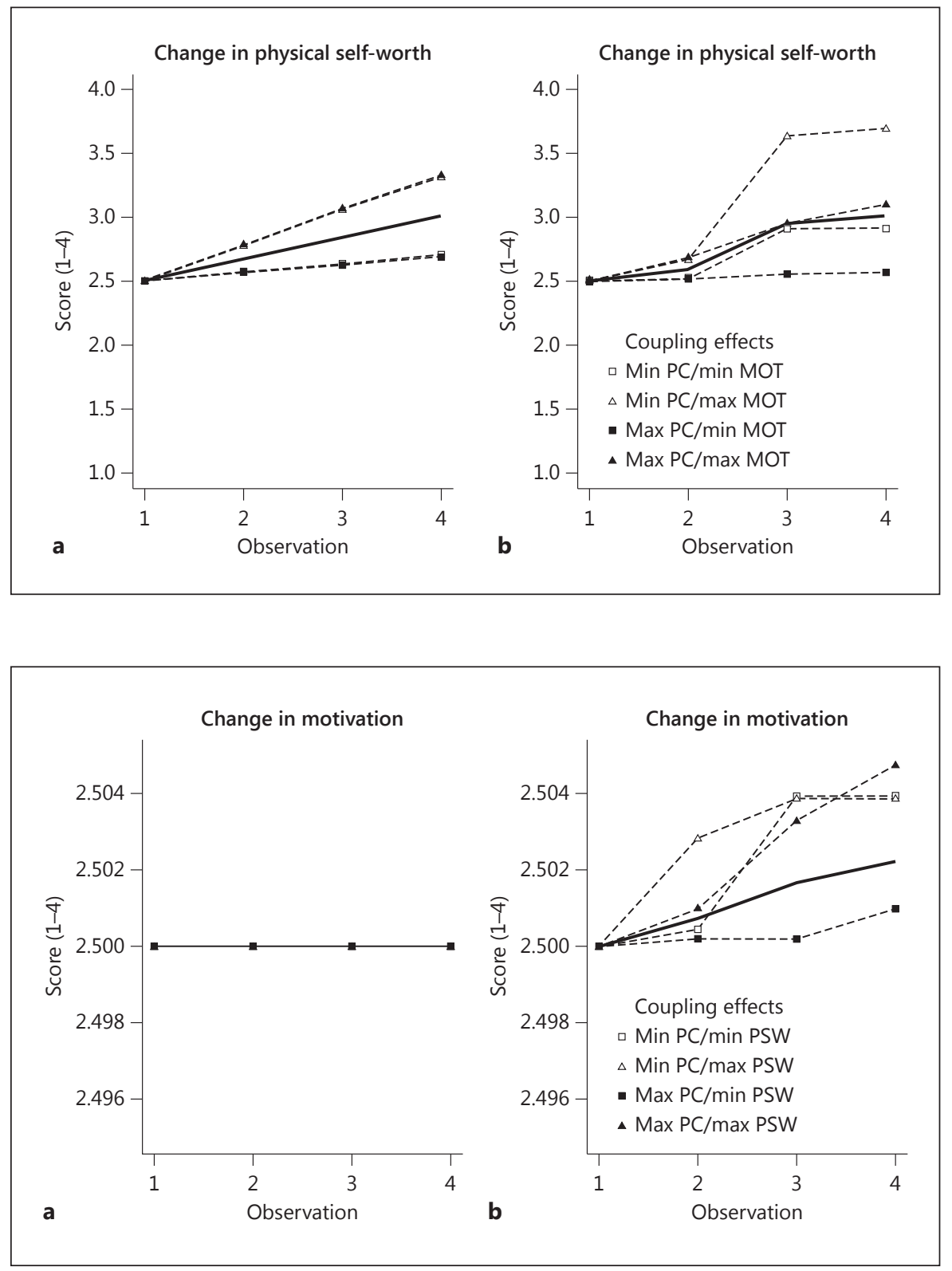

Fig. 10. Expected latent trajectories for motivation as a function of varying initial scores (minimum, average and maximum) for perceived competence (PC) and physical self-worth (PSW) based on the first (a) and second (b) multivariate model. ized clinical trials [20], models involving multiple groups [21], multiple processes [16, 17], latent variables with measurement structure $[2,8]$ as well as more complex nonlinear processes [7].

As described in the introduction, these LCS models are applicable to research questions consistent with the objectives of longitudinal methodology [1]. In our illustration, we examined the dynamics of a network of processes. The results from this analytic approach provided information about sources underlying changes in the system. The coupling parameters in these LCS models represent the relation of one process at a given occasion with changes in another process at a subsequent time point. These effects can be interpreted as developmental sequences in which current states predict future changes, thus mapping onto Baltes and Nesselroade's [1] last two objectives.

While any SEM computer program can be used to conduct analyses using LCS models, a number of steps are required. First, longitudinal data are necessary to detect within-person changes and, thus, to model latent changes that pertain to each person. Such longitudinal data can be 
in the form of panel data on multiple variables and few occasions, or with longer time series. Second, LCS models assume discrete intervals with equal spacing, as first difference models. This requirement, however, can be relaxed, as specifications of LCS models exist that assume continuous time [22-24]. Third, LCS models rely on mathematical and statistical theories from SEM with latent variables. Issues of invariance and fit are relevant, as are those regarding measurement properties (i.e. reliability, sample sizes and power) to identify changes and individual differences in growth curves $[25,26]$. Fourth, LCS models serve to define implied latent changes in multiple variables, and such changes are then predicted using dynamic theory.

One particularly appealing feature of LCS models is that they combine the identification of changes in multiple processes with the underlying dynamics among them. Because of this, LCS models are helpful to evaluate hypotheses that involve interrelations among various processes together with changes in those processes over time. Additionally, unlike some autoregressive and crosslagged models (e.g. simplex and quasi-simplex models), LCS models model the changes directly and incorporate a slope parameter to describe an unknown, latent, mean change process at each measurement occasion, with a variance component representing differences in this effect across subjects. Therefore, this model is not only useful for accounting for lagged relations between processes, but also for mean changes and variability in such changes over time (see Ferrer and McArlde [4] and McArdle [3] for a description of such differences).

Another important feature of LCS models is the detection of sequences among variables over time. This is par- ticularly relevant when examining developmental processes that do not occur in a continuous fashion and that could exhibit spurts. In these instances, important goals of the analyses are identifying points of developmental discontinuities as well variables related to such rupture points. Some attempts to capture nonlinearities have consisted of time segments, specifying different dynamics across time intervals $[16,27]$. This is, no doubt, limited, and a more parsimonious approach would require the detection of, say, the number and location of segments throughout the developmental course, possible transitions across the various segments as well as nonlinear specifications that can account for the nonlinearity and the dynamics [7].

In sum, we present a flexible SEM approach to analyze multivariate longitudinal data, and a brief demonstration of the applications of such models to empirical data. By illustrating some of the possibilities of LCS modeling for studying changes and dynamics in multivariate systems, we hope to promote further exploration and utilization of these models in future research.

\section{Acknowledgments}

This work was supported in part by grants from the National Science Foundation (BCS-05-27766 and BCS-08-27021) and NIHNINDS (R01 NS057146-01) to Emilio Ferrer.

\section{Disclosure Statement}

The authors declare that they do not have any conflict of interest in this work.

\section{References}

1 Baltes PB, Nesselroade JR: History and rationale of longitudinal research; in Nesselroade JR, Baltes PB (eds): Longitudinal Research in the Study of Behavior and Development. New York, Academic Press, 1979, pp 1-40.

2 McArdle JJ: A latent difference score approach to longitudinal dynamic structural analysis; in Cudeck R, du Toit S, Sörbom D (eds): Structural Equation Modeling: Present and Future. A Festschrift in Honor of Karl Jöreskog. Lincolnwood, Scientific Software International, 2001, pp 341-380.

3 McArdle JJ: Latent variable modeling of differences in changes with longitudinal data. Annu Rev Psychol 2009;60:577-605.
-4 Ferrer E, McArdle JJ: Longitudinal modeling of developmental changes in psychological research. Curr Dir Psychol Sci 2010;19:149154.

$>5$ McArdle JJ, Hamagami F: Linear dynamic analyses of incomplete longitudinal data; in Collins L, Sayer A (eds): New Methods for the Analysis of Change. Washington, APA, 2001, pp 137-176.

-6 Ferrer E, McArdle JJ: Alternative structural models for multivariate longitudinal data analysis. Struct Equ Model 2003;10:493-524.

7 Hamagami F, McArdle JJ: Dynamic extensions of latent difference score models; in Boker SM, Wegner ML (eds): Quantitative Methods in Contemporary Psychology. Mahwah, Erlbaum, 2007, pp 47-85.
$>8$ Ferrer E, Balluerka N, Widaman KF: Factorial invariance and the specification of second-order latent growth models. Methodology 2008;4:22-36.

$\checkmark 9$ Harter S: The development of self-representations; in Eisenberg N (ed): Handbook of Child Psychology. New York, Wiley, 1998, vol 3: Social, Emotional, and Personality Development, pp 553-618.

10 Whitehead JR: A study of children's self-perceptions using an adapted physical self-perception profile questionnaire. Pediatr Exerc Sci 1995;7:132-151.

11 Stinnett TA, Oehler-Stinnett J, Stout LJ: Development of the teacher rating of academic achievement motivation: TRAAM. School Psychol Rev 1991;20:609-622. 
12 Harter S: The determinants and mediational role of global self-worth in children; in Eisenberg N (ed): Contemporary Issues in Developmental Psychology. New York, Wiley, 1987, pp 219-242.

13 Harter S: The Construction of the Self: A Developmental Perspective. New York, Guilford, 1999.

14 Deci EL, Ryan RM: Intrinsic Motivation and Self-Determination in Human Behavior. New York, Plenum, 1985.

15 Deci EL, Vallerand RJ, Pelletier LG, Ryan RM: Motivation and education: the self-determination perspective. Educ Psychol 1991;26: 325-346.

16 Ferrer E, McArdle JJ: An experimental analysis of dynamic hypotheses about cognitive abilities and achievement from childhood to early adulthood. Dev Psychol 2004;40:935952.

17 McArdle JJ, Hamagami F, Meredith W, Bradway KP: Modeling the dynamic hypotheses of Gf-Gc theory using longitudinal life-span data. Learn Individ Diff 2000;12:53-79.
18 Hamagami F, McArdle JJ, Nesselroade JR, Ferrer E, Boker S: Modeling Acceleration of Dynamic Systems with Interindividual Variation Using Structured Difference Equation Methodology. Technical Report. Charlottesville, University of Virginia, 2003.

19 Grimm KJ, An Y, McArdle JJ, Zonderman $\mathrm{AB}$, Resnick SM: Recent changes leading to subsequent changes: extensions of multivariate latent difference score models. Struct Equ Model 2012;19:268-292.

20 McArdle JJ, Prindle JJ: A latent change score analysis of a randomized clinical trial in reasoning training. Psychol Aging 2008;23:702719.

21 Ferrer E, Shaywitz BA, Holahan JN, Marchione K, Shaywitz SE: Uncoupling of reading and IQ over time: empirical evidence for a definition of dyslexia. Psychol Sci 2010;21: 93-101.

22 Oud JHL, Delsing MJMH: Continuous time modeling of panel data by means of SEM; in van Montfort K, Oud JHL, Satorra A (eds): Longitudinal Research with Latent Variables. New York, Springer, 2010, pp 201-244.
23 Voelkle MC, Oud JHL: Continuous time modelling with individually varying time intervals for oscillating and non-oscillating processes. Br J Math Stat Psychol 2013;66:103126.

24 Voelkle MC, Oud JHL, Davidov E, Schmidt P: An SEM approach to continuous time modeling of panel data: relating authoritarianism and anomia. Psychol Methods 2012;17:176192.

25 Hertzog C, Lindenberger U, Ghisletta P, von Oertzen T: On the power of multivariate latent growth curve models to detect correlated change. Psychol Methods 2006;11:244-252.

26 Hertzog C, von Oertzen T, Ghisletta P, Lindenberger U: Evaluating the power of latent growth curve models to detect individual differences in change. Struct Equ Model 2008; 15 : 541-563.

27 Ferrer E, McArdle JJ, Shaywitz BA, Holahan JN, Marchione K, Shaywitz SE: Longitudinal models of developmental dynamics between reading and cognition from childhood to adolescence. Dev Psychol 2007;43:1460-1473. 